\title{
Cost-Effectiveness Analysis of Rivaroxaban Plus Aspirin Compared with Aspirin Alone in Patients with Coronary and Peripheral Artery Diseases in Italy
}

\author{
Pietro Ferrara ${ }^{1,2}$ (D) Paolo A. Cortesi ${ }^{1,2} \cdot$ Danilo Di Laura $^{1} \cdot$ Aldo P. Maggioni ${ }^{3,4} \cdot$ Lorenzo G. Mantovani $^{1,2}$
}

Accepted: 6 March 2021 / Published online: 16 March 2021

(c) The Author(s) 2021

\begin{abstract}
Background Rivaroxaban is a selective inhibitor of coagulation factor Xa and its combination with aspirin showed better outcomes in the prevention of recurrent cardiovascular disease than aspirin alone.

Objective This analysis aimed to economically compare the cost effectiveness of rivaroxaban ( $2.5 \mathrm{mg}$ twice daily) plus aspirin (100 mg once daily) with aspirin alone in patients with coronary artery disease (CAD) or peripheral artery disease (PAD) and related subgroups.

Methods The analysis simulates the perspective of the Italian National Healthcare Service and used a state-transition decision Markov model. Clinical efficacy data and health events risks were gathered from the COMPASS trial. Health outcomes and costs (in Euros) were evaluated over a lifetime horizon and were discounted at 3.5\% per annum. Direct healthcare costs entered the analysis. Results were expressed in terms of incremental cost-effectiveness ratio (ICER), defined as cost per quality-adjusted life-year (QALY) gained. One-way deterministic and probabilistic sensitivity analyses were performed.

Results For the CAD or PAD population, rivaroxaban plus aspirin was more effective and costly compared with aspirin alone. Incremental costs and efficacy produced an ICER of $€ 16,522$ per QALY gained. Analyses found similar trends for the PAD and CAD groups, with respective ICERs of $€ 8003$ and $€ 18,599$, while ICERs for the other groups were lower than $€ 13,000$ per QALY. Sensitivity analyses confirmed these findings.

Conclusion Compared with aspirin alone, rivaroxaban plus aspirin is cost effective in preventing recurrent cardiovascular events in all patients with CAD or PAD, from the Italian perspective. These results could help clinicians and decision makers to develop improved strategies for cardiovascular disease prevention.
\end{abstract}

Pietro Ferrara

p.ferrara5@campus.unimib.it

1 Center for Public Health Research, University of MilanoBicocca, Via Cadore 48, 20900 Monza, Italy

2 Value-Based Healthcare Unit, IRCCS MultiMedica, Sesto San Giovanni, Italy

3 ANMCO Research Center, Florence, Italy

4 Maria Cecilia Hospital, GVM Care and Research, Cotignola, RA, Italy

\section{Key Points}

Low-dose rivaroxaban and aspirin improve cardiovascular outcomes in subjects at risk of major adverse cardiovascular events.

Compared with aspirin alone, rivaroxaban plus aspirin is cost effective in preventing recurrent cardiovascular events in all patients with coronary artery disease or peripheral artery disease.

Cost effectiveness is also confirmed from the perspective of the Italian National Healthcare Service. 


\section{Introduction}

Cardiovascular diseases (CVDs), such as ischemic heart disease and stroke, are the principal causes of death and one of the leading causes of disability in high-income countries $[1,2]$. Individuals with coronary artery disease (CAD) or peripheral artery disease (PAD) constitute a large proportion of patients at high risk of atherothrombotic cardiovascular events and death. In these populations, the role of antiplatelet therapy with aspirin as an anchor strategy for secondary cardiovascular prevention is largely recognized, with a $19 \%$ lower risk of major adverse cardiovascular events (MACE) and a 9\% lower risk of cardiovascular death than placebo [3].

Rivaroxaban is a selective direct inhibitor of the factor $\mathrm{Xa}$ of coagulation that has been approved for the prevention and treatment of venous thromboembolism (VTE), as well as the prevention of stroke and systemic embolism in atrial fibrillation [4-6]. The Cardiovascular Outcomes for People Using Anti-coagulation Strategies (COMPASS) study, defined as a double-blind superiority trial conducted in 33 countries, including Italy, showed that the combination of low-dose rivaroxaban $2.5 \mathrm{mg}$ twice daily and aspirin significantly improved cardiovascular outcomes than aspirin alone, with a respective $24 \%$ and $18 \%$ relative risk reduction of MACE, such as myocardial infarction (MI), ischemic stroke (IS), or cardiovascular death, as well as all-cause death [7].

Available evidence underlined antiplatelet therapy cost effectiveness in the prevention of these conditions [8,9]; however, there are few evidences on the cost effectiveness of rivaroxaban plus aspirin in secondary prevention among CAD or PAD populations and subgroups. To our knowledge, the cost effectiveness of this combination in the European context has been assessed in the UK and The Netherlands $[10,11]$, however no study has been conducted from the Italian perspective. Outside Europe, more analyses were conducted from the Australian societal and healthcare perspective and the Canadian healthcare perspective [12-14]. In this context, it should be remarked that the pharmaceutical market of Italy is one of the largest worldwide and in Europe and is often fixed as the marketing authorization benchmark [15].

Thus, the objective of this work was to conduct a costeffectiveness evaluation, in patients with CAD or PAD, of rivaroxaban $2.5 \mathrm{mg}$ twice daily in combination with aspirin $100 \mathrm{mg}$ once daily compared with aspirin $100 \mathrm{mg}$ once daily alone as the primary comparison, over a lifetime horizon, from the Italian National Healthcare Service (NHS) perspective.

\section{Materials and Methods}

\subsection{Model Overview}

A published state-transition decision analytic Markov model, which gathered clinical data from the COMPASS trial, was used to evaluate the cost effectiveness of rivaroxaban plus aspirin compared with aspirin alone in patients with CAD or PAD [10]. This economic framework was adapted to simulate the perspective of the Italian NHS, over a lifetime horizon timeframe and considering a discount rate of 3.5\% per annum for future costs and outcomes.

\subsection{Model Structure}

The schematic Markov model is presented in Fig. 1. The model assumes that patients can be classified into a determinate number of mutually exclusive health states, expressed by disease parameters. A patient can transit from one state to another. Briefly, patients with CAD or PAD may enter the model in an event-free state and remain in this state or experience one event among MI, IS, intracranial hemorrhage (ICH), a combination of these, or death. After the first event, patients move to a post-acute health state and/ or experience a second event. Furthermore, the following

Fig. 1 Markov model diagram. $M I$ myocardial infarction, $I S$ ischemic stroke, $\mathrm{ICH}$ intracranial hemorrhage

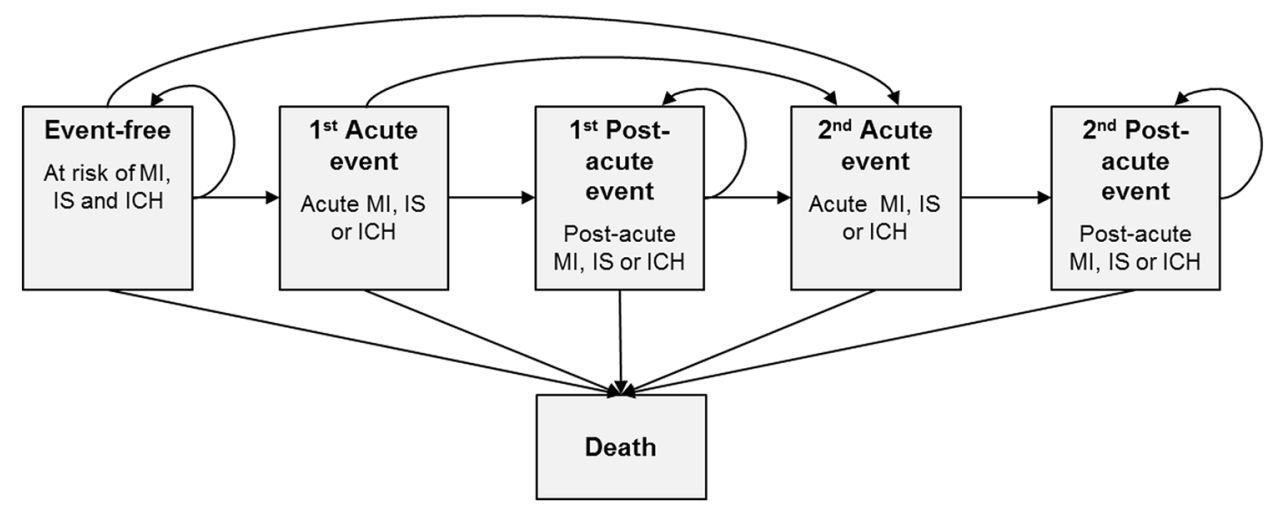


health events assessed in the COMPASS trial are accounted for in the model: major amputation, major extracranial nonfatal bleed, acute limb ischemia (ALI), minor amputation, and VTE. These health events could occur during simulation in all health states. Finally, the model also accounted for the gender-level background mortality of the Italian population on the basis of the last available statistics regarding death from the Italian National Statistics Institute (ISTAT) [16], in order to account for male and female prevalence within simulated populations. From these background data, the proportions of deaths attributable to CVD were removed to avoid double counting.

\subsection{Model Population}

All patients entering the Markov model were derived from the COMPASS study, which included subjects who met the criteria for CAD, PAD, or both, from 33 countries (including Italy) [7]. CAD patients aged $<65$ years were included if they had documented atherosclerosis involving at least two vascular beds or at least two additional risk factors (such as current smoking, diabetes mellitus, an estimated glomerular filtration rate $<60 \mathrm{~mL} / \mathrm{min}$, heart failure [HF], or non-lacunar IS $\geq 1$ month earlier). The entire population was therefore stratified into six subgroups: CAD or PAD, CAD (irrespective of PAD status), PAD (irrespective of CAD status), $\mathrm{CAD}$ and PAD, CAD with chronic kidney disease (CKD), and $\mathrm{CAD}$ with HF. We considered all population subgroups for the purpose of this study. The overall characteristics of these populations have been described elsewhere [7], while age and gender distribution are presented in Table 1.

\subsection{Clinical Inputs}

Since the study population was derived from the COMPASS trial, the proposed analytic model integrated baseline patient characteristics, clinical input parameters, and transition probabilities sourced using patient-level data from the same study [7], which are presented in Table 1 (and detailed in electronic supplementary Table S1). These include fatal and non-fatal events, for both the first event in event-free patients and second events in those with a one-event history at the 3 -month period (Table 1). Transition probabilities were estimated by performing a survival analysis for each event, both in the entire population and in each specific subgroup, using COMPASS patient-level data. Furthermore, since the treatment effect of rivaroxaban plus aspirin was consistent across all subgroups included in the COMPASS trial, the hazard ratio (HR) for each considered clinical event included in the analyses was that of the overall CAD or PAD population of the COMPASS trial, which was applied to all subpopulations considered in this study. A similar discontinuation probability was assumed for rivaroxaban plus aspirin (3-month probability $=0.0287)$ and aspirin alone (3-month probability $=0.0268$ ) in all simulated groups, based on the results of the COMPASS trial [7].

\subsection{Cost Inputs}

All costs are reported in Euros $(€)$ and refer to the year 2020. Since the model was built from the Italian healthcare perspective, only direct health costs were included in the analysis. Each health event accounted for in the economic model, along with its cost and source, is listed in Table 2. Costs in the third month for the main health events were calculated on the basis of evidence-based annual costs for the considered health events [17-20]. The Italian national tariffs were used for estimating costs of ICH, ALI, minor amputation, major non-fatal extracranial bleed, and VTE on the basis of 2013 Diagnosis-Related Group (DRG) tariffs [17]; event-related tariffs were multiplied for the respective event risks listed in Table 1 (and detailed in electronic supplementary Table S1). Following a more conservative approach, costs associated with cardiovascular death were not included in the analysis. Drug prices for aspirin were obtained from the Italian Agency of Medicine (AIFA) tariffs ( $€ 1.41$ for 30 tablets of $100 \mathrm{mg}$ ) [21]. At the time of the analysis, rivaroxaban was not reimbursed for CAD or PAD treatment; we assumed the same treatment cost ( $€ 2.09$ per day) of rivaroxaban dosage $(10,15$, and $20 \mathrm{mg})$ approved for other clinical conditions and based on the ex-factory price including the statutory discounts as per Italian legislation ( $€ 58.52$ for 56 tablets of $2.5 \mathrm{mg}$ ) [22].

\subsection{Utilities}

Utility data were estimated using the EuroQol-5D tool (EQ-5D) from the COMPASS analysis. There, the baseline EQ-5D weight for the CAD or PAD population was 0.835 for the event-free health state. Utility decrements for health states and events were calculated through multivariate regression analysis by adjusting for age, gender and baseline EQ-5D value. Decrements were assumed to be the same for all populations included in the analysis and for all treatment arms, as there was no evidence to suggest that treatment choice had any impact on quality of life. In addition, for second health events, the utility for those patients was considered to be the lowest utility of the individual included in the health states. The utility weight of the main health events ( $0-3$ months) included in the model, as well as utility decrements considered in the base case for health events, are illustrated in Table 1 (and detailed in electronic supplementary Table S1). 
Table 1 Clinical data input from the COMPASS trial

\begin{tabular}{|c|c|c|c|c|c|c|}
\hline Parameter & $\mathrm{CAD}$ or $\mathrm{PAD}$ & $\mathrm{PAD}$ & CAD & $\mathrm{CAD}$ and $\mathrm{PAD}$ & CAD with CKD & CAD with $\mathrm{HF}$ \\
\hline Age, years [mean (range)] & $68.24(60-75)$ & $67.81(60-75)$ & $68.34(60-75)$ & $68.06(60-75)$ & $71.74(65-79)$ & $65.49(58-72)$ \\
\hline Sex, male $[n(\%)]$ & $\begin{array}{l}21,375 / 27,395 \\
(78.0)\end{array}$ & $\begin{array}{l}5361 / 7470 \\
(71.8)\end{array}$ & $\begin{array}{l}19,792 / 24,824 \\
(79.7)\end{array}$ & $\begin{array}{l}3782 / 4906 \\
(77.1)\end{array}$ & $\begin{array}{l}3038 / 5561 \\
(70.8)\end{array}$ & $\begin{array}{l}4418 / 5714 \\
(77.3)\end{array}$ \\
\hline \multicolumn{7}{|l|}{ Clinical inputs } \\
\hline \multicolumn{7}{|c|}{ Risk of acute main events (fatal and non-fatal, ASA arm), 3-month probability } \\
\hline MI & 0.00290 & 0.00357 & 0.00301 & 0.00448 & 0.00483 & 0.00361 \\
\hline IS & 0.00176 & 0.00225 & 0.00174 & 0.00236 & 0.00256 & 0.00250 \\
\hline $\mathrm{ICH}$ & 0.00029 & 0.00049 & 0.00030 & 0.00065 & 0.00043 & 0.00015 \\
\hline ALI & 0.00064 & 0.00221 & 0.00043 & 0.00191 & 0.00069 & 0.00072 \\
\hline VTE & 0.00061 & 0.00092 & 0.00062 & 0.00111 & 0.00104 & 0.00072 \\
\hline Major extracranial bleed & 0.00217 & 0.00226 & 0.00219 & 0.00239 & 0.00367 & 0.00239 \\
\hline Minor amputation & 0.00043 & 0.00135 & 0.00026 & 0.00096 & 0.00055 & 0.00036 \\
\hline Major amputation & 0.00037 & 0.00124 & 0.00019 & 0.00072 & 0.00041 & 0.00029 \\
\hline \multicolumn{7}{|c|}{ RIV + ASA treatment efficacy in terms of events (relative to ASA alone) } \\
\hline & $\mathrm{HR}(95 \% \mathrm{CI})$ & & & & & \\
\hline MI & $0.86(0.70-1.05)$ & & & & & \\
\hline IS & $0.51(0.38-0.69)$ & & & & & \\
\hline $\mathrm{ICH}$ & $1.16(0.67-2.00)$ & & & & & \\
\hline ALI & $0.55(0.32-0.93)$ & & & & & \\
\hline VTE & $0.61(0.37-1.00)$ & & & & & \\
\hline Major extracranial bleed & $1.79(1.46-2.19)$ & & & & & \\
\hline Minor amputation & $0.65(0.35-1.20)$ & & & & & \\
\hline Major amputation & $0.57(0.30-1.09)$ & & & & & \\
\hline \multicolumn{7}{|c|}{ Utility weight of events ( $0-3$ months), mean } \\
\hline MI & 0.7840 & 0.7390 & 0.7910 & 0.7470 & 0.7630 & 0.7510 \\
\hline IS & 0.6470 & 0.6100 & 0.6520 & 0.6170 & 0.6300 & 0.6200 \\
\hline $\mathrm{ICH}$ & 0.7020 & 0.6620 & 0.7080 & 0.6690 & 0.6840 & 0.6730 \\
\hline \multicolumn{7}{|c|}{ Utility decrements for health events } \\
\hline ALI & -0.157 & & & & & \\
\hline VTE & -0.111 & & & & & \\
\hline Major extracranial bleed & -0.019 & & & & & \\
\hline Minor amputation & -0.100 & & & & & \\
\hline Major amputation & -0.175 & & & & & \\
\hline
\end{tabular}

Data inputs are derived from the COMPASS trial [7]

$C A D$ coronary artery disease, $P A D$ peripheral artery disease, $C K D$ chronic kidney disease, $H F$ heart failure, $R I V$ rivaroxaban, $A S A$ aspirin, $M I$ myocardial infarction, $I S$ ischemic stroke, $I C H$ intracranial hemorrhage, $H R$ hazard ratio, $C I$ confidence interval, $A L I$ acute limb ischemia, VTE venous thromboembolism

\subsection{Analysis}

The model estimated the costs $(€)$ and quality-adjusted lifeyears (QALYs) obtained for each simulated treatment. It also assessed the specific costs associated with treatments and the costs associated with other healthcare resources. The cost effectiveness of rivaroxaban plus aspirin versus aspirin alone was evaluated in terms of the incremental cost-effectiveness ratio (ICER), calculated by dividing the difference in cost between two treatment arms by the difference in QALYs gained and considering a societal willingness-to-pay (WTP) threshold of $€ 40,000$ per QALY [23]. The lifetime treatment duration of rivaroxaban plus aspirin or aspirin alone was consistent with the expected label in the population of interest, on the basis of treatment guidelines.

\subsection{Sensitivity Analysis}

Validation of the model parameters was performed using deterministic sensitivity analyses (DSA) and probabilistic sensitivity analyses (PSA). The DSA was conducted to assess the impact of individual parameters on the results. 
Table 2 Model cost inputs

\begin{tabular}{lll}
\hline Parameter & Value (in Euros, $€$ ) & Source \\
\hline Medication & & \\
Aspirin (100 mg once daily) & $1.41^{\mathrm{a}}$ & AIFA \\
Rivaroxaban (2.5 mg twice daily) & $58.52^{\mathrm{b}}$ & AIFA \\
Health costs & & \\
Acute myocardial infarction (0-3 months) & 7545 & Mantovani et al., 2011 [18] \\
Post-acute myocardial infarction (3+months) & 980 & Mantovani et al., 2011 [18] \\
Acute stroke (0-3 months) & 6164 & Lucchese et al., 2017 [19] \\
Post-acute stroke (3+months) & 1855 & Lucchese et al., 2017 [19] \\
Intracranial hemorrhage & 3891 & DRG no. 14 [17] \\
Acute limb ischemia & 2199 & DRG no. 130, 131 [17] \\
Minor amputation & 6056 & DRG no. 114 [17] \\
Major amputation (0-3 months) & 13,948 & Marone et al., 2018 [20] \\
Post-major amputation (3+months) & 4375 & Marone et al., 2018 [20] \\
Major non-fatal extracranial bleed & 2091 & DRG no. 175 [17] \\
Venous thromboembolism & 2315 & DRG no. 128 [17]
\end{tabular}

AIFA Italian Agency of Medicine, $D R G$ Italian diagnosis-related group tariff

${ }^{\text {a }}$ Pack price (30 tablets) - AIFA

${ }^{\mathrm{b}}$ Pack price (56 tablets) ex-factory plus statutory discounts $(-5 \%,-5 \%)$

Variables with a major input to the costs were identified and the results presented in the Tornado diagrams. Furthermore, an extensive DSA was performed to assess the impact for rivaroxaban price due to the lack of reimbursed price for PAD or CAD patients. In the PSA, built to evaluate the joint uncertainty in the model parameters on the costeffectiveness results, the ICER was recalculated 1000 times through Monte Carlo simulations, while all input variables were varied simultaneously over their distribution. In addition, specific scenarios on treatment discontinuation and persistence were also considered. First, patients treated with rivaroxaban plus aspirin were assumed to switch to aspirin alone after 5 years. Another two scenarios studied treatment persistence (1) over 4 years, assuming that no patient discontinued, and (2) considering a lifetime discontinuation rate with an impact on both efficacy and costs. Analyses were conducted using Microsoft Excel ${ }^{\circledR}$ (Microsoft Corporation, Redmond, WA USA; 2016) [24].

\section{Results}

\subsection{Main Results}

Accounting for the entire $\mathrm{CAD}$ or $\mathrm{PAD}$ population, rivaroxaban in combination with aspirin was more effective than aspirin alone in terms of QALYs, with average QALYs of 9.62 and 9.27 for patients in the two groups, respectively. Rivaroxaban plus aspirin reported higher lifetime treatment costs partially offset by the reduction of other healthcare costs, with an incremental cost per patient of $€ 5785$. The incremental costs and efficacy (QALY) associated with rivaroxaban plus aspirin produced an ICER of $€ 16,522$ per QALY gained.

Rivaroxaban plus aspirin was found to be more effective compared with aspirin alone in all populations studied, being significantly below the WTP threshold of $€ 40,000$ per QALY gained. Table 3 and electronic supplementary Fig. S1 illustrate the results of the cost-effectiveness analyses. The analyses found similar trends for the PAD and CAD subgroups, with respective ICERs of $€ 8003$ and $€ 18,599$, both being below the fixed WTP threshold. In the CAD and PAD, CAD with CKD, and CAD with HF subgroups, ICERs were lower than $€ 13,000$ per QALY gained.

\subsection{Sensitivity Analysis}

The one-way DSA showed that the combination of rivaroxaban plus aspirin remained cost effective in all tested alternative scenarios, with ICERs remaining below $€ 30,000$ per QALY. The most sensitive parameters were age and treatment efficacy in terms of the main health event rates. For the PAD subgroup, ICER was also sensitive to 3-month risk and cost duration of major amputation. The results according to each studied population are presented in the Tornado diagrams shown in Fig. 2. In the $\mathrm{CAD}$ or PAD, PAD, and CAD PSAs, rivaroxaban plus aspirin was cost effective in $96.6 \%, 100 \%$, and $92.8 \%$ of the simulations, respectively, compared with aspirin alone at a WTP threshold of $€ 30,000$ per QALY gained (Fig. 3). The probability rose to $100 \%$ in all subpopulations using 
Table 3 Cost-effectiveness analysis results

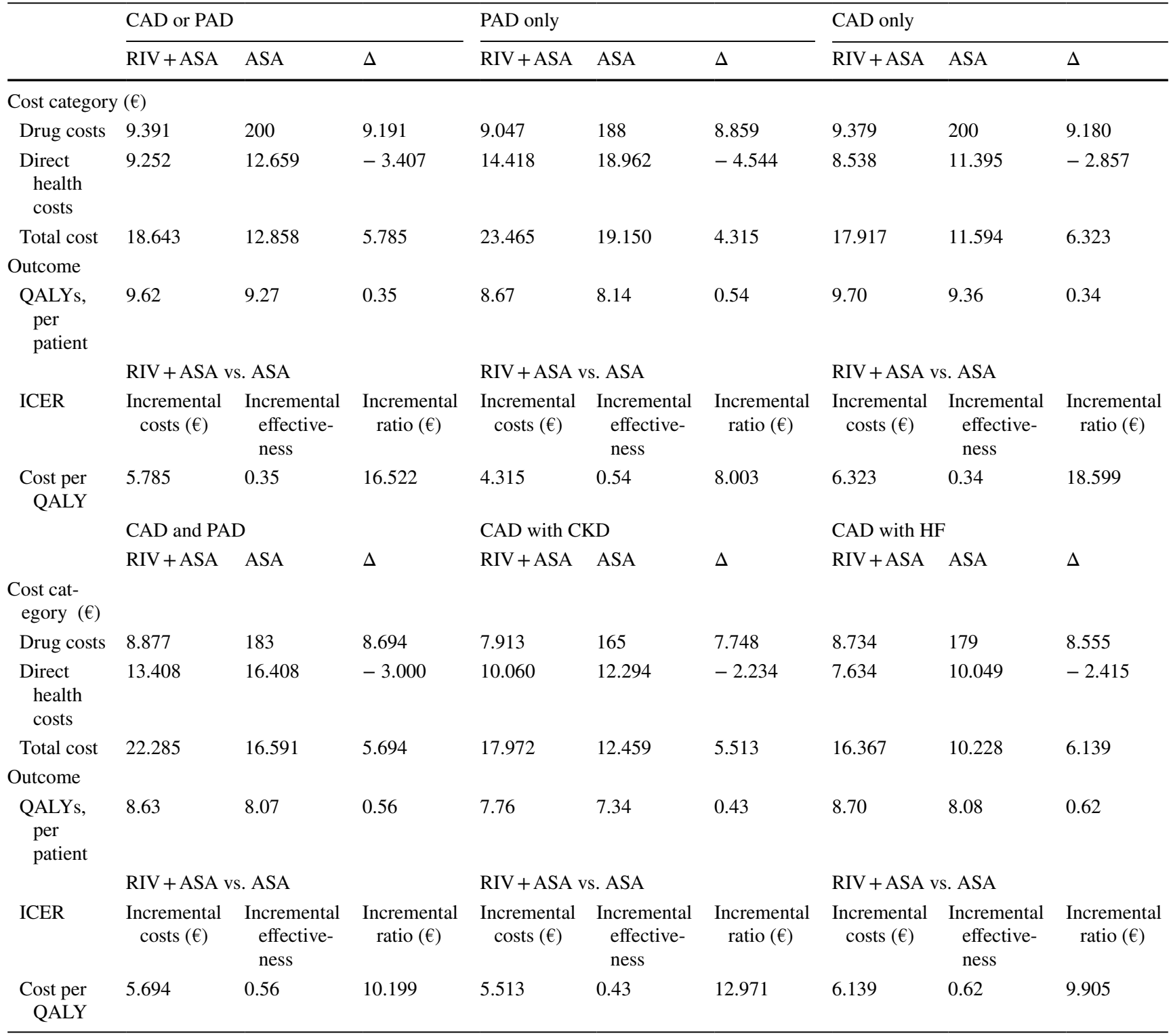

All costs are reported in Euros $(€)$

Delta symbol defines the difference between information (costs, QALYs and ICERs) for RIV+ASA vs. ASA alone

$C A D$ coronary artery disease, $P A D$ peripheral artery disease, $C K D$ chronic kidney disease, $H F$ heart failure, $R I V$ rivaroxaban, $A S A$ aspirin, $Q A L Y$ quality-adjusted life-year, ICER incremental cost-effectiveness ratio

a WTP threshold of $€ 40,000$ per QALY gained (Fig. 3 and electronic supplementary Fig. S1). Overall DSA and PSA results are presented in Fig. 2 and Fig. 3. Analyses on treatment duration showed a reduction of incremental costs. A 5-year treatment duration for rivaroxaban plus aspirin resulted in a reduction in the ICER by $13 \%$. With regard to treatment persistence scenarios, significant reductions in the ICERs were observed, by $51 \%$ and $29 \%$, respectively, depending on the scenario. In rivaroxaban price sensitivity analysis, with a $30 \%$ price decrease, an ICER reduction nearing 50\% (approximately €8000 per QALY gained) was seen in the CAD or PAD population. In the PAD and CAD subgroups, a $40 \%$ decrease in the rivaroxaban price showed an ICER reduction higher than $50 \%$. Similarly, for the other three subgroups analyzed (CAD and PAD, CAD with CKD, and CAD with HF), a $50 \%$ ICER reduction was observed with a price reduction of $20 \%$. Detailed results are reported in Fig. 4. 


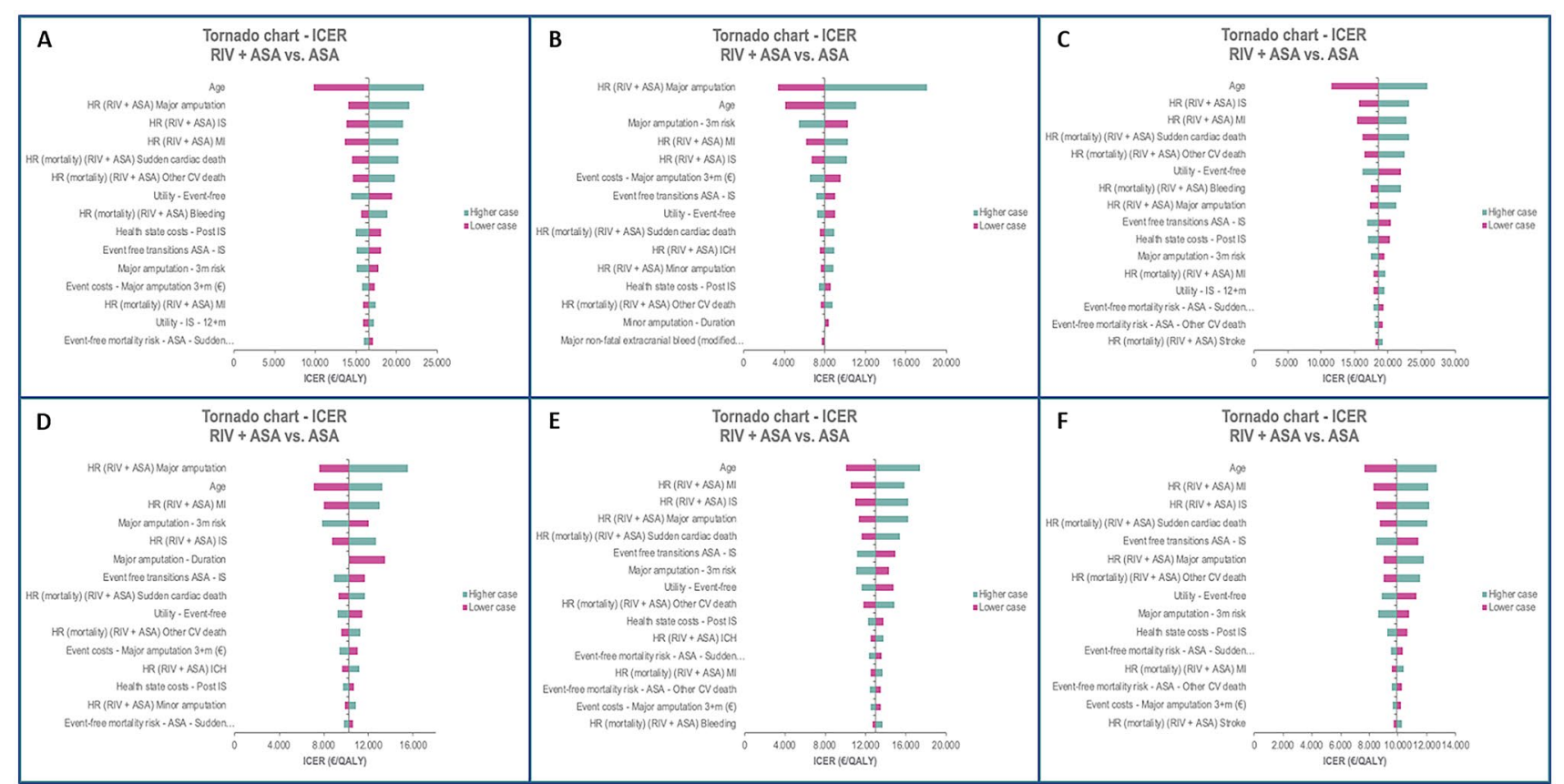

Fig. 2 Tornado chart of the one-way deterministic analyses. Populations included are $\mathbf{a}$ CAD or PAD, b PAD, $\mathbf{c}$ CAD, $\mathbf{d}$ CAD and PAD, e CAD with CKD, and $\mathbf{f}$ CAD with HF. Pink bar indicates that the low value of the parameter has been used, and the green bar indicates that the high value of the parameter has been used. $H R$ hazard ratio,
$C A D$ coronary artery disease, $P A D$ peripheral artery disease, $C K D$ chronic kidney disease, $H F$ heart failure, $R I V$ rivaroxaban, $A S A$ aspirin, $Q A L Y$ quality-adjusted life-year, ICER incremental cost-effectiveness ratio, $M I$ myocardial infarction, $I S$ ischemic stroke, $C V$ cardiovascular, $\mathrm{ICH}$ intracranial hemorrhage

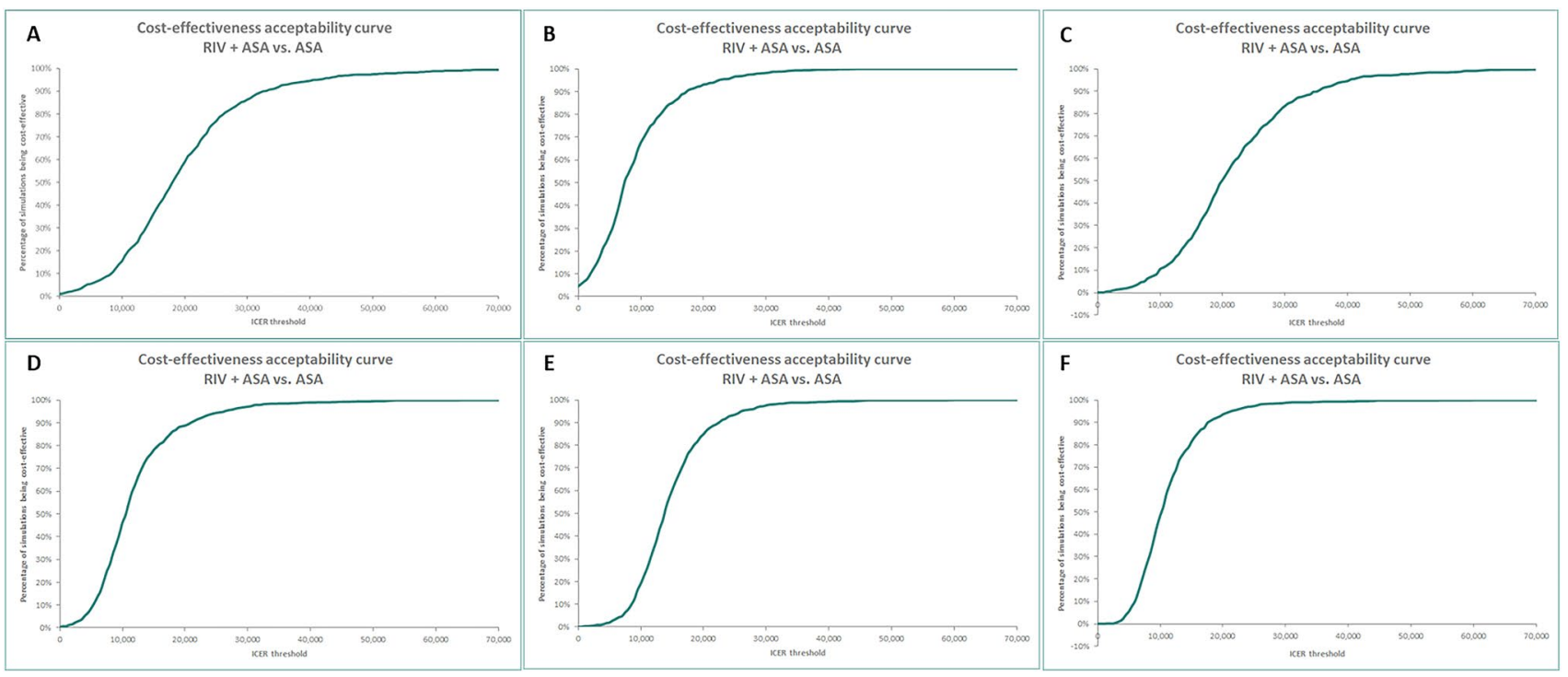

Fig. 3 Cost-effectiveness acceptability curve according to Monte Carlo simulations with 1000 iterations. Populations included are a CAD or PAD, b PAD, $\mathbf{c} C A D, \mathbf{d} C A D$ and PAD, e CAD with CKD, and $\mathbf{f}$ CAD with HF. $C A D$ coronary artery disease, $P A D$ peripheral artery disease, $C K D$ chronic kidney disease, $H F$ heart failure, $R I V$ rivaroxaban, $A S A$ aspirin, ICER incremental cost-effectiveness ratio 


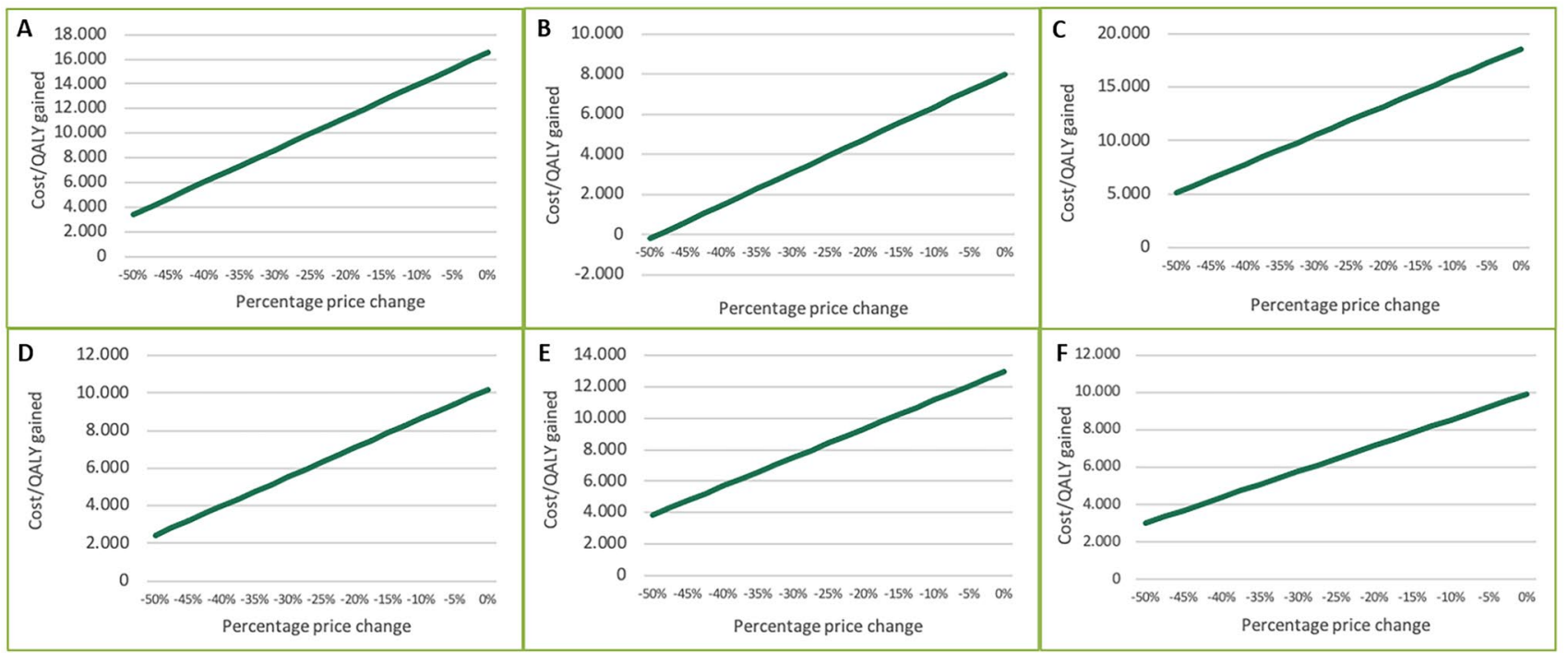

Fig. 4 Cost-effectiveness price threshold analysis. Populations included are a CAD or PAD, b PAD, $\mathbf{c}$ CAD, $\mathbf{d}$ CAD and PAD, e $\mathrm{CAD}$ with $\mathrm{CKD}$, and $\mathbf{f} \mathrm{CAD}$ with $\mathrm{HF}$. $C A D$ coronary artery disease,

\section{Discussion}

This analysis aimed to evaluate the cost effectiveness of rivaroxaban in combination with aspirin compared with aspirin alone in patients with vascular disease, from the perspective of the Italian public healthcare system. The first important finding is that this drug combination was found to be cost effective in all populations included in the analysis. In addition, this cost effectiveness is also maintained across different scenarios. In particular, the cost effectiveness of rivaroxaban plus aspirin is clearly evident in all subgroups of patients, but especially in those at higher risk, such as patients with PAD or those with CAD with HF. The results of the one-way DSA showed that the main factors impacting on the ICER were treatment efficacy in terms of main health event rates and 3-month risk, age, and cost duration of major amputation. The PSA showed that compared with aspirin alone, the combination of rivaroxaban with aspirin was cost effective in almost all simulations, at the WTP threshold of $€ 40,000$ per QALY gained, with $90 \%$ of simulations below $€ 25,000$ per QALY gained. To confirm and improve the reliability of the presented results, further research should study more in-depth parameters that affect these results on the basis of DSA.

The overall cost effectiveness is robustly sustained by the superiority of rivaroxaban with aspirin versus aspirin alone in terms of health outcomes, causing a significant reduction of health expenditure and cost duration attributable to costly health events such as amputations, MIs, and stroke, and their follow-up care.
$P A D$ peripheral artery disease, $C K D$ chronic kidney disease, $H F$ heart failure, $Q A L Y$ quality-adjusted life-year

These findings mirrored previous economic analyses of rivaroxaban plus aspirin compared with aspirin alone in patients with CAD or PAD conducted in other countries such as Australia, Canada, The Netherlands, and the UK [10-14]. For instance, the two researches conducted from the Australian perspective provided strong evidence that rivaroxaban in combination with aspirin was cost effective compared with aspirin alone in the prevention of recurrent CVD events and major adverse limb events in populations of patients with CAD or PAD $[12,13]$. These analyses were built using COMPASS transition probabilities through a Markov model with annual cycles consisting of three health states: alive with PAD or CAD and no recurrent CVD, alive with PAD or CAD and recurrent CVD, and death. The study reported an ICER of AU $\$ 26,769$ per QALY over a lifetime horizon [13] and AU\$31,436 per QALY over 20-year time horizon [12]. Similarly, the Canadian Agency for Drugs and Technologies in Health confirmed such findings, concluding that rivaroxaban was cost effective in patients with concomitant $\mathrm{CAD}$ and PAD, assuming the same conditions as our economic framework and resulting in an ICER per QALY gained of \$17,764 [14]. For the European context, we retrieved similar findings in the analysis conducted from the UK NHS perspective for the CAD or PAD population. In this analysis, rivaroxaban plus aspirin reported an ICER of $£ 16,360$ per QALY gained compared with aspirin alone, resulting in a cost-effective option for the UK NHS [10]. This result was confirmed by the PSA with rivaroxaban plus aspirin resulted cost-effective in $98 \%$ of simulation at a WTP threshold of $£ 30,000$ per QALY. Lastly, the Dutch analysis reported ICERs for the comparison of the studied dual pathway 
inhibition versus aspirin alone of $€ 32,109$ and $€ 26,381$ in $C A D$ and PAD patients, respectively [11].

Compared with the other mentioned cost-effectiveness studies [10-14], the strength of our research is the analysis based on patient-level data from the COMPASS study, as already described, of all subgroups derived from the trial: CAD or PAD, CAD, PAD, CAD and PAD, CAD with CKD, and CAD with HF. To the best of our knowledge, no other published studies have completely analyzed all of these subgroups, with their specific health event risk retrieved from the aspirin arm of the COMPASS trial. In fact, except for a secondary analysis in the UK NHS setting that considered patients with CAD (irrespective of PAD status) and PAD (irrespective of CAD status) without detailing the data input and the results [10], available cost-effectiveness evidence was limited to only all CAD and/or PAD populations [11-14]. Thus, our study added further information on the cost effectiveness of rivaroxaban plus aspirin in all subgroups, showing specific cost-effectiveness profiles for each subgroup. In fact, we estimated the ICERs of CAD ( $€ 18,599$ per QALY gained), PAD ( $€ 8003), C A D$ and PAD $(€ 10,199)$, CAD with CKD $(€ 12,971)$, and CAD with HF (€9905), reporting rivaroxaban plus aspirin as the cost-effectiveness option in each single subgroup.

As such, the detailing of cost effectiveness in all COMPASS subpopulations makes our results suitable for other NHS settings in other countries, including those where research was limited to the overall CAD and PAD population. Furthermore, in light of the last evidence of the COMPASS trial, which suggests overlapping benefits between diabetic and non-diabetic patients in terms of relative risk reduction, but a higher absolute risk reduction in diabetic patients, rivaroxaban plus aspirin has the potential to also be even more cost effective in this higher-risk population [20, 25]. Therefore, new and specific cost-effectiveness evaluations are required in the near future to assess the value of rivaroxaban plus aspirin in the $\mathrm{CAD}$ and/or PAD diabetic subgroups. Similarly, direct comparative effectiveness studies should be performed in the near future to compare the therapeutic strategy analyzed with possible alternatives for the prevention of recurrent cardiovascular events, such as the combination of aspirin and clopidogrel.

Finally, the analyses showed the cost effectiveness of rivaroxaban (2.5 $\mathrm{mg}$ twice daily) plus aspirin (100 $\mathrm{mg}$ once daily) compared with the current standard of care from the Italian health payer perspective, assuming a daily cost of $€ 2.09$. A possible lower price of rivaroxaban $2.5 \mathrm{mg}$ twice daily could only improve the cost effectiveness of this treatment in all subgroups, as shown in the ICER variations according to the percentage price reduction (Fig. 4).

Even if the present analysis was based on methodological standards, it should be acknowledged that this study has a number of limitations that must be taken into account when interpreting the results. First, the analysis included data on rivaroxaban in combination with aspirin compared with aspirin alone sourced from the COMPASS trial on relatively short-term (23-month period) data, which were assumed to remain equal over a lifetime horizon. This was limited as a real-world study, due to a possible decrease in benefits over time that could affect cost effectiveness. Sensitivity analyses should have mitigated this limitation exploring the impact of age variability and reporting ICERs significantly below the WTP threshold of $€ 40,000$ per QALY gained. Second, the analysis was based on the COMPASS population. As a trial population, this might be different from a real-world population; however, the real-world population is generally at higher risk for health events, and rivaroxaban plus aspirin could be even more cost-effective. Furthermore, the safety profile could be different using rivaroxaban plus aspirin in the real-word population and this analysis must be updated as soon as real-word data on a longer follow-up period are available. Third, the use of DRG tariffs as a proxy parameter for modeling costs could lead to the limitation of cost-effectiveness analyses under the common condition of insufficient or unavailable data from hospital-level cost studies. Nevertheless, the DRG tariff appears to a be a rigid estimate for hospital expenses $[19,26]$.

Despite these limitations, the methodology of the study is accurate and it provides reliable findings confirmed by sensitivity analyses.

\section{Conclusion}

The present research highlights that, compared with aspirin alone, rivaroxaban $2.5 \mathrm{mg}$ twice daily in combination with aspirin $100 \mathrm{mg}$ once daily is cost effective in preventing recurrent cardiovascular events in patients with $\mathrm{CAD}$ and PAD, from the Italian healthcare perspective. Our study reported on the cost effectiveness of rivaroxaban plus aspirin in the entire COMPASS population (CAD or PAD), as well as in all five subpopulations (CAD, PAD, CAD and PAD, CAD with HF, and CAD with CKD), based on the specific health event risk and relative treatment impact. These results need to be confirmed by observational studies on rivaroxaban use for CAD or PAD in the real-word patient population in clinical practice.

Supplementary Information The online version contains supplementary material available at https://doi.org/10.1007/s40261-021-01023-8.

\section{Declarations}

Funding Open access funding provided by Università degli Studi di Milano - Bicocca within the CRUI-CARE Agreement. 
Conflicts of Interest Pietro Ferrara, Paolo A. Cortesi, Danilo Di Laura, Aldo P. Maggioni, and Lorenzo G. Mantovani have no conflicts of interest to disclose.

Ethics Approval This study did not involve participants as information was gathered from previously published research and freely accessible public databases; thus, no ethical approval was required.

Consent to Participate Not applicable.

Consent for Publication Not applicable.

Code Availability Not applicable.

Author Contributions All authors contributed to the conception or design of the work and the acquisition, analysis and interpretation of data, and drafted and critically revised the manuscript. All authors gave final approval and agree to be accountable for all aspects of the work, ensuring integrity and accuracy.

Open Access This article is licensed under a Creative Commons Attribution-NonCommercial 4.0 International License, which permits any non-commercial use, sharing, adaptation, distribution and reproduction in any medium or format, as long as you give appropriate credit to the original author(s) and the source, provide a link to the Creative Commons licence, and indicate if changes were made. The images or other third party material in this article are included in the article's Creative Commons licence, unless indicated otherwise in a credit line to the material. If material is not included in the article's Creative Commons licence and your intended use is not permitted by statutory regulation or exceeds the permitted use, you will need to obtain permission directly from the copyright holder. To view a copy of this licence, visit http://creativecommons.org/licenses/by-nc/4.0/.

\section{References}

1. GBD 2019 Diseases and Injuries Collaborators. Global burden of 369 diseases and injuries in 204 countries and territories, 1990-2019: a systematic analysis for the Global Burden of Disease Study 2019. Lancet. 2020;396(10258):1204-22.

2. Roth GA, Mensah GA, Johnson CO, et al. Global burden of cardiovascular diseases and risk factors, 1990 to 2019: update from the global burden of disease 2019 study. J Am Coll Cardiol. 2020;76(25):2982-3021. https://doi.org/10.1016/j.jacc.2020.11.010.

3. Antithrombotic Trialists' (ATT) Collaboration. Aspirin in the primary and secondary prevention of vascular disease: collaborative meta-analysis of individual participant data from randomised trials. Lancet. 2009;373:1849-60.

4. The EINSTEIN-PE Investigators. Oral rivaroxaban for the treatment of symptomatic pulmonary embolism. N Engl J Med. 2012;366:1287-97.

5. The EINSTEIN Investigators. Oral rivaroxaban for symptomatic venous thromboembolism. N Engl J Med. 2010;363:2499-510.

6. Patel MR, Mahaffey KW, Garg J, et al. Rivaroxaban versus warfarin in nonvalvular atrial fibrillation. N Engl J Med. 2011;365:883-91.

7. Eikelboom JW, Connolly SJ, Bosch J, et al. Rivaroxaban with or without aspirin in stable cardiovascular disease. N Engl J Med. 2017;377(14):1319-30.

8. Lopez-Lopez JA, Sterne JAC, Thom HHZ, et al. Oral anticoagulants for prevention of stroke in atrial fibrillation: systematic review, network meta-analysis, and cost effectiveness analysis. BMJ. 2017;359:
9. Sterne JA, Bodalia PN, Bryden PA, et al. Oral anticoagulants for primary prevention, treatment and secondary prevention of venous thromboembolic disease, and for prevention of stroke in atrial fibrillation: systematic review, network meta-analysis and costeffectiveness analysis. Health Technol Assess. 2017;21(9):1-386.

10. Cowie MR, Lamy A, Levy P, Mealing S, Millier A, Mernagh P, Cristeau O, Bowrin K, Briere JB. Health economic evaluation of rivaroxaban in the treatment of patients with chronic coronary artery disease or peripheral artery disease. Cardiovasc Res. 2020;116(11):1918-24.

11. Petersohn S, Pouwels X, Ramaekers B, Ten Cate-Hoek A, Joore M. Rivaroxaban plus aspirin for the prevention of ischaemic events in patients with cardiovascular disease: a cost-effectiveness study. Eur J Prev Cardiol. 2020;27(13):1354-65.

12. Ademi Z, Zomer E, Tonkin A, Liew D. Cost-effectiveness of rivaroxaban and aspirin compared to aspirin alone in patients with stable cardiovascular disease: an Australian perspective. Int J Cardiol. 2018;270:54-9.

13. Zomer E, Si S, Hird TR, et al. Cost-effectiveness of low-dose rivaroxaban and aspirin versus aspirin alone in people with peripheral or carotid artery disease: an Australian healthcare perspective. Eur J Prev Cardiol. 2019;26(8):858-68.

14. Canadian Agency for Drugs and Technologies in Health (CADTH). Common Drug Review-Pharmacoeconomic Review Report for Rivaroxaban (Xarelto). CADTH; 2019.

15. Panteli D, Arickx F, Cleemput I, et al. Pharmaceutical regulation in 15 European countries: review. Health Syst Trans. 2016;18(5):1-118.

16. Istituto nazionale di statistica (ISTAT). I.Stat database. http://dati. istat.it. Accessed 9 Feb 2021.

17. Italian Diagnosis-Related Group (DRG). Decreto del Ministero della Salute 18 ottobre 2012. In: Gazzetta Ufficiale della Repubblica Italiana n. 23 del 28 gennaio 2013.

18. Mantovani LG, Fornari C, Madotto F, et al. Burden of acute myocardial infarction. Int J Cardiol. 2011;150(1):111-2.

19. Lucchese M, Borisenko O, Mantovani LG, et al. Cost-utility analysis of bariatric surgery in italy: results of decision-analytic modelling. Obes Facts. 2017;10(3):261-72.

20. Marone EM, Cozzolino P, Ciampichini R, et al. Peripheral arterial disease in diabetic patients: a long-term population-based study on occurrence, outcomes and cost. J Cardiovasc Surg (Torino). 2018;59(4):572-9.

21. Agenzia italiana del farmaco (AIFA). Liste di trasparenza e rimborsabilità. http://www.agenziafarmaco.gov.it/content/liste-ditrasparenza-e-rimborsabilita. Accessed 9 Feb 2021.

22. Agenzia italiana del farmaco (AIFA). Determina del 27 settembre 2006-Manovra per il governo della spesa farmaceutica convenzionata e non convenzionata. https://www.gazzettaufficiale.it/atto/ serie_generale/caricaDettaglioAtto/originario?atto.dataPubbli cazioneGazzetta=2006-09-29\&atto.codiceRedazionale $=06$ A08 826\&elenco30giorni=false. Accessed 9 Feb 2021.

23. Cortesi PA, Belli LS Facchetti R, et al. The optimal timing of hepatitis $\mathrm{C}$ therapy in liver transplant-eligible patients: costeffectiveness analysis of new opportunities. J Viral Hepat. 2018;25(7):791-801.

24. Microsoft Excel ${ }^{\circledR}$ (Software). Microsoft Office Professional Plus 2016. Redmond: Microsoft Corporation; 2016. p. 2016.

25. Bhatt DL, Eikelboom JW, Connolly SJ, et al. The role of combination antiplatelet and anticoagulation therapy in diabetes and cardiovascular disease: insights from the COMPASS trial. Circulation. 2020;141(23):1841-54. https://doi.org/10.1161/CIRCU LATIONAHA.120.046448.

26. Glick HA, Doshi JA, Sonnad SS, Polsky D. Economic evaluation in clinical trials. Oxford: Oxford University Press; 2015. 